\title{
Remediation and Cognitive Enhancers in Schizotypal Personality Disorder
}

Fiona S. Graff, PsyD $D^{1, *}$

Margaret M. McClure, PhD $D^{1,2}$

Larry J. Siever, $M D^{1}$

\author{
Address \\ ${ }^{*}, 1$ VA VISN3 Mental Illness, Research, Education, and Clinical Center (MIRECC)/ \\ Icahn School of Medicine at Mount Sinai, James J. Peters VA Medical Center, 130 \\ West Kingsbridge Road, Bronx, NY 10468, USA \\ e-mail: fiona.graff@mssm.edu \\ e-mail: larry.siever@va.gov \\ ${ }^{2}$ Psychology Department, Fairfield University, 1073 North Benson Road, Fairfield, \\ CT 06824, USA \\ e-mail: margaret.mcnamara@mssm.edu
}

Published online: 14 0ctober 2014

(C) Springer International Publishing AG (outside the USA) 2014

Keywords Schizotypal personality disorder · Cognitive impairment - Remediation

\section{Opinion statement}

Individuals with schizotypal personality disorder (SPD) often present with cognitive impairment similar, but of a lesser magnitude to, what is seen in schizophrenia. Cognitive dysfunction combined with social and perceptual disturbances, which are the hallmarks of this disorder, are directly related to substantially lower functioning. There is no FDAapproved medication for cognitive deficits in SPD; however, potential agents, including a2a and dopamine agonists, are under investigation. Cognitive remediation therapy (CRT) is a behavioral treatment that has been shown to improve cognitive and psychosocial functioning in individuals with schizophrenia. Application of CRT in this population is novel and more large-scale studies are needed, but this treatment holds promise for the cognitive and social deficits of SPD. There may be a synergy between cognitive remediation and cognitive enhancers such as the $\alpha 2 a$ agonist guanfacine; research in this area is nascent. Lifestyle modifications such as the use of memory aids, planners, and structured schedules, as well as exercise, may be beneficial for cognitive functioning in this population. Additionally, social skills training in a psychotherapeutic setting can be helpful. Patients frequently present with comorbid mood and anxiety symptoms, and standard SSRI treatment to ameliorate symptoms can have a positive secondary effect on cognitive functioning. For the patient with psychotic-like features, treatment with atypical antipsychotics may also improve overall functioning. 


\section{Introduction}

Schizotypal personality disorder (SPD) is classified in DSM-5 within the category of "Schizophrenia Spectrum and Other Psychotic Disorders," and is characterized by significant social deficits and discomfort, cognitive difficulties and perceptual disturbances, paranoia, and unusual behavior [1]. SPD shares genetic links with schizophrenia [2] and frequently clusters in families of individuals with schizophrenia [3]. The prevalence of SPD is estimated at approximately $3.9 \%$ and is slightly more common among males than females [4]. SPD is highly comorbid with mood (44\%), anxiety (57\%), and substance use disorders (44\%), as well as with other personality disorders (83\%), particularly cluster B diagnoses [4].

Individuals with SPD frequently manifest significant impairment in functioning: these persons often realize limited occupational status $[5 \bullet, 6,7]$, reduced mental health, and social and emotional difficulties [4]. Cognitive deficits in this population are notable, particularly in working memory [8•] and context processing [9]. In imaging studies, these individuals display temporal lobe volume reductions similar to schizophrenia [10], yet frontal lobe volume appears to be largely intact or increased $[10,11]$. This has contributed to the hypothesis that compensatory frontal lobe functioning protects these individuals from the degree of psychosis and impairment seen in schizophrenia $[3,8 \bullet 10]$.
Patients typically present to psychiatric treatment with complaints of mood and anxiety symptoms rather than with concerns about cognitive, social and occupational functioning per se [1]. Literature regarding psychosocial and psychiatric treatment of this disorder is limited. However, in one treatment utilization study, patients with SPD were more likely to receive individual therapy, inpatient hospitalization, and treatment in a halfway house than those with a depression diagnosis [12], likely reflecting the significant extent of functional impairment. Depressive symptoms are treated with antidepressant therapy and antipsychotics may also be prescribed for attendant psychotic-like symptoms. While there is no pharmacotherapy specifically indicated for the cognitive deficits of SPD and large-scale replication studies of investigational agents are needed, a trial of the $\alpha 2 \mathrm{a}$ agonist guanfacine has shown promise as a cognitive enhancing agent [13]. Other mechanisms explored in preliminary studies include dopamine agonists [14, 15] and an anticholinesterase inhibitor [16]. Research on psychosocial interventions for SPD is scarce; however, cognitive remediation therapy (CRT) combined with social skills training for the cognitive and social deficits in this population is a promising avenue under investigation.

There are several excellent reviews of the etiology, and functional and cognitive impairment of SPD, which are outlined below in the section on References and Recommended Reading.

\section{Treatment}

- Several lifestyle adjustments can help with cognitive difficulties experienced in SPD:

- Employ memory aids such as planners, calendars and reminders.

- Some individuals find that development and maintenance of a structured schedule is helpful in improving cognitive function and managing symptoms.

- Exercise has been shown to increase hippocampal volume in individuals with schizophrenia [17, Class II] as well as increase brain-derived neurotrophic factor (BDNF), a substance associated with neural plasticity [18], which could facilitate learning and memory. 
- Evidence suggests that malnutrition may be associated with reduced cognitive status and subsequent development of SPD traits [19] and as such, adequate nutrition is important. Omega-3 dietary supplements may be an additional area for research based on promising findings from the schizophrenia literature [20].

- Individuals with SPD may be encouraged to develop and access social outlets at community locations including libraries, religious centers, senior/community centers, etc. Some individuals may benefit from learning to test out paranoid assumptions and interpretations of others' behavior. This is likely to be most effective within the context of an ongoing therapeutic relationship with a mental health professional.

- Significant comorbid mood and anxiety symptoms can directly affect cognitive performance and should be managed through psychosocial and/or pharmacological intervention (e.g., [21, Class IV]).

- Mood, anxiety, and psychotic symptoms can contribute to cognitive impairment; as such, pharmacologic treatment according to standards of practice may be warranted. Low-dose atypical antipsychotics are sometimes prescribed and may help improve functioning [22, Class I].

- A small study reported positive and negative symptom reduction with a 9-week trial of a low-dose (titrated to $2.0 \mathrm{mg} /$ day) of risperidone [7, Class II]; however, a 12-week trial of risperidone (up to $2.0 \mathrm{mg} /$ day) versus placebo in SPD was not associated with improvement in cognitive outcome [23, Class II].

- There is preliminary evidence supporting further exploration of dopamine and $\alpha 2$ a agonists as pharmacotherapies for cognitive deficits in SPD. All trials are investigational and no pharmacotherapies specifically for cognitive enhancement are used in routine clinical practice at this time.

\section{$\alpha 2$ adrenergic agonists}

\section{Guanfacine}

In a double-blind randomized trial of 29 patients with SPD, individuals receiving four weeks of the $\alpha 2 \mathrm{a}$ agonist guanfacine demonstrated improvement in context processing, or the ability to accurately use previously presented information in behavioral responses [13, Class I].

Standard procedure Dosage was titrated to $2.0 \mathrm{mg} /$ day.

Contraindications

As guanfacine is an antihypertensive, contraindications are clinically significant for cardiovascular or neurological conditions including uncontrolled 
hypertension, arrhythmias, angina, ventricular hypertrophy, clinically significant EKG abnormalities, or concurrent antihypertensive medication.

Complications None observed.

Special points Large-scale replication trials are needed.

Cost/cost-effectiveness N/A

\section{Dopamine agonists}

\section{Pergolide}

Twenty-five patients with SPD were randomized to pergolide, a $\mathrm{D}_{1}$ and $\mathrm{D}_{2}$ agonist, versus placebo, for four weeks. Those in the active condition demonstrated improvement on verbal memory, verbal working memory, and executive functioning [15, Class I].

Standard procedure Patients in the active condition were titrated up to $0.3 \mathrm{mg} / \mathrm{day}$.

Contraindications Cardiac valve problems

Complications/Special points During the course of this study, FDA approval for pergolide was revoked secondary to concerns about increased risk of valvular heart damage. This trial's enrollment was suspended due to safety concerns of ergot-derived dopamine agonists, although no participants experienced adverse events.

Cost/cost-effectiveness N/A

\section{Amphetamine}
Standard procedure
Contraindications
$30 \mathrm{mg}$ amphetamine, administered orally, in investigational challenges.
Includes cardiac or vascular disease or hypertension, hyperthyroidism, stimu- lant sensitivity, and a history of substance abuse. In addition, amphetamine is contraindicated in individuals currently or recently administered monoamine oxidase inhibitors (MAOIs).
Complications/Special points N/A
Cost/cost-effectiveness N/A

In two separate randomized double-blind trials, individuals with SPD undergoing a d-amphetamine challenge demonstrated reduced perseveration errors in a test of problem-solving [14, Class I], as well as greater improvement versus the comparison group on visuospatial memory [24, Class I].

Cognitive remediation therapy (CRT) is a behavioral intervention designed to improve cognition through repeated stimulation of areas important in cognitive functioning (see expert's workshop definition, [25]). Treatment involves repeated and massed delivery of training exercises across a range of cognitive domains and is frequently delivered on the computer and in group formats. CRT is associated with positive cognitive and functional outcome in schizophrenia [26•]. CRT is frequently paired with a skills-training or a vocational rehabilitation group, with the goal of increasing real-world generalization of computer skills. The 
application of CRT to SPD is conceptually sound given its shared etiology and holds promise as a potential intervention for cognitive and functional deficits in SPD, but, as yet, there are no trials of effectiveness.

Standard procedure

Our group is currently testing a trial of cognitive remediation and social skills training in SPD. The trial is ongoing; however, preliminary results are promising in terms of participant tolerability and response.

In our experimental procedure, participants receive 7.5 weeks of twiceweekly computerized, coach-facilitated cognitive remediation treatment, plus concurrent social skills training. Computerized sessions employ Cognitive Enhancement Therapy [27], and participants complete cognitive training exercises in small groups. Administration in small groups encourages additional opportunity for social skills practice, a particularly important exercise for this often socially isolated group of individuals. The addition of social skills training follows from schizophrenia research literature suggesting that transfer of learning is most salient when computer training is paired with a skills group [28]. In these groups, topics relevant to both cognitive difficulties (e.g., ways to enhance memory) and interpersonal issues (e.g., assessing and testing assumptions around nonverbal cues) are emphasized. We have developed our group curriculum based on the CET manual of Hogarty \& Greenwald (2006), which is a companion to Psychological Software Services training sessions.

Contraindications Our group has not encountered any contraindications; cognitive training exercises are accessible, and even those individuals with limited computer facility can develop confidence and engage in the exercises.

Complications Group training may be an aversive activity to some individuals, particularly those presenting with significant paranoia and suspiciousness. In our experience, following a well-structured and explicit agenda in social skills training has been helpful in fostering comfort and reducing anxiety.

Special points We are also testing the addition of guanfacine to cognitive remediation to explore whether these two treatments are synergistic. In a small, randomized sample $(n=8)$, the combined approach outperformed cognitive remediation alone in processing speed and problem solving (unpublished data), although replication is necessary.

Cost/cost-effectiveness Cognitive-remediation therapy is a relatively costly program. Participants attend treatment twice weekly and cognitive remediation coaching and social skills sessions are led by program staff. In addition, there are material costs of computers, space, and software.

\section{Conclusion}

Schizotypal personality disorder (SPD) is a personality disorder marked by substantial cognitive and social impairment. Individuals with this diagnosis demonstrate structural and cognitive deficits similar to those experienced by individuals with schizophrenia, have a high rate of psychiatric comorbidity, and typically realize reduced functioning. Cognitive deficits in areas such as working 
memory and context processing are notable and contribute to functional difficulties. Because some of the hallmark symptoms of this disorder are paranoia and social isolation, individuals with SPD may be reluctant to seek treatment for SPD symptoms; however, these individuals may present for co-occurring mood and anxiety symptoms. While there are no FDA-approved pharmacological agents used in routine clinical care for cognitive deficits experienced by patients with SPD, agents under investigation include $\alpha 2 \mathrm{a}$ and dopamine agonists. Lifestyle modifications such as memory aids, exercise, and adequate diet may be helpful. Psychotherapeutic interventions include treating anxiety and depression, which may be exacerbating cognitive difficulties and encouraging increased social interaction for some patients. Additionally, cognitive remediation therapy plus social skills training, which has demonstrated benefits in schizophrenia, is a promising avenue currently under investigation.

\section{Compliance with Ethics Guidelines}

\section{Conflict of Interest}

Fiona S. Graff, Margaret M. McClure, and Larry J. Siever declare no conflicts of interest.

\section{Human and Animal Rights and Informed Consent}

This article includes studies with human subjects performed by the authors. All studies had Institutional Review Board approval, and Informed Consent was obtained from all participants.

\section{References and Recommended Reading}

Papers of particular interest, published recently, have been highlighted as:

- Of importance

1. American Psychiatric Association. Diagnostic and statistical manual of mental disorders. 5th ed. Arlington: American Psychiatric Publishing; 2013.

2. Tienari P, Wynne LC, Laksy K, et al. Genetic boundaries of the schizophrenia spectrum: evidence from the Finnish adoptive family study of schizophrenia. Am J Psychiatry. 2003;160(9):1587-94.

3. Siever LJ, Davis KL. The pathophysiology of schizophrenia disorders: perspectives from the spectrum. Am J Psychiatry. 2004;161(3):398-413.

4. Pulay AJ, Stinson FS, Dawson DA, et al. Prevalence, correlates, disability, and comorbidity of DSM-IV schizotypal personality disorder: results from the wave 2 national epidemiologic survey on alcohol and related conditions. Prim Care Companion J Clin Psychiatry. 2009;11(2):53-67.

5. $\quad$ McClure MM, Harvey PD, Bowie CR, Iacoviello B, Siever LJ. Functional outcomes, functional capacity, and cognitive impairment in schizotypal personality disorder. Schizophr Res. 2013;144(1-3):146-50.
This article highlights the significant functional impairment frequently experienced by individuals with SPD

6. McGurk SR, Mueser KT, Mischel R, et al. Vocational functioning in schizotypal and paranoid personality disorders. Psychiatry Res. 2013;210(2):498-504.

7. Koenigsberg HW, Reynolds D, Goodman M, et al. Risperidone in the treatment of schizotypal personality disorder. J Clin Psychiatry. 2003;64(6):628-34.

8. Hazlett EA, Lamade RV, Graff FS, et al. Visual-spatial working memory performance and temporal gray matter volume predict schizotypal personality disorder group membership. Schizophr Res. 2014;152(2-3):350-7.

This article demonstrates the particular salience of working memory deficits in SPD

9. McClure MM, Barch DM, Flory JD, Harvey PD, Siever LJ. Context processing in schizotypal personality disorder: evidence of specificity of impairment to the schizophrenia spectrum. J Abnorm Psychol. 2008;117(2):342-54. 
10. Hazlett EA, Buchsbaum MS, Haznedar MM, et al. Cortical gray and white matter volume in unmedicated schizotypal and schizophrenia patients. Schizophr Res. 2008;101(1-3):111-23.

11. Kuhn S, Schubert F, Gallinat J. Higher prefrontal cortical thickness in high schizotypal personality trait. J Psychiatr Res. 2012;46(7):960-5.

12. Bender DS, Dolan RT, Skodol AE, et al. Treatment utilization by patients with personality disorders. Am J Psychiatry. 2001;158(2):295-302.

13. McClure MM, Barch DM, Romero MJ, et al. The effects of guanfacine on context processing abnormalities in schizotypal personality disorder. Biol Psychiatry. 2007;61(10):1157-60.

14. Siegel Jr BV, Trestman RL, O'Flaithbheartaigh S, et al. Damphetamine challenge effects on Wisconsin Card Sort Test performance in schizotypal personality disorder. Schizophr Res. 1996;20(1-2):29-32.

15. McClure MM, Harvey PD, Goodman M, et al. Pergolide treatment of cognitive deficits associated with schizotypal personality disorder: continued evidence of the importance of the dopamine system in the schizophrenia spectrum. Neuropsychopharmacology. 2010;35(6):1356-62.

16. Steinberg BJ, Trestman R, Mitropoulou V, et al. Depressive response to physostigmine challenge in borderline personality disorder patients.

Neuropsychopharmacology. 1997;17(4):264-73.

17. Pajonk FG, Wobrock T, Gruber O, et al. Hippocampal plasticity in response to exercise in schizophrenia. Arch Gen Psychiatry. 2010;67(2):133-43.

18. Cotman CW, Berchtold NC. Exercise: a behavioral intervention to enhance brain health and plasticity. Trends Neurosci. 2002;25(6):295-301.

19. Venables PH, Raine A. Poor nutrition at age 3 and schizotypal personality at age 23 : the mediating role of age 11 cognitive functioning. Am J Psychiatry. 2012;169(8):822-30.

20. Amminger G, Schäfer MR, Papageorgiou K, et al. Longchain $\omega-3$ fatty acids for indicated prevention of psychotic disorders: a randomized, placebo-controlled trial. Arch Gen Psychiatry. 2010;67(2):146-54.

21. Nathanson BJ, Jamison SC. Psychotherapeutic and pharmacologic treatment of schizotypal personality disorder: the heuristic utility of stressing function over form. Clin Case Stud. 2011.

22. Ripoll LH, Triebwasser J, Siever LJ. Evidence-based pharmacotherapy for personality disorders. Int J Neuropsychopharmacol. 2011;14(9):1257-88.

23. McClure MM, Koenigsberg HW, Reynolds D, et al. The effects of risperidone on the cognitive performance of individuals with schizotypal personality disorder. J Clin Psychopharmacol. 2009;29(4):396-8.

24. Kirrane RM, Mitropoulou V, Nunn M, et al. Effects of amphetamine on visuospatial working memory performance in schizophrenia spectrum personality disorder. Neuropsychopharmacology. 2000;22(1):14-8.

25. Wykes T, Spaulding WD. Thinking about the future cognitive remediation therapy-what works and could we do better? Schizophr Bull. 2011;37 Suppl 2:S80-90.

26. Wykes T, Huddy V, Cellard C, McGurk SR, Czobor P. A meta-analysis of cognitive remediation for schizophrenia: methodology and effect sizes. Am J Psychiatry. 2011;168(5):472-85.

This is an overview of cognitive remediation therapy and metaanalytic data supporting its effectiveness

27. Hogarty GE, Greenwald DP. Cognitive enhancement therapy: the training manual. CET Training: Pittsburgh; 2006.

28. McGurk SR, Twamley EW, Sitzer DI, McHugo GJ, Mueser KT. A meta-analysis of cognitive remediation in schizophrenia. Am J Psychiatry. 2007;164(12):1791802. 